\title{
HOLISMO E INVESTIGACIÓN CUALITATIVA EN EL MARCO DE LA ANTROPOLOGÍA DE LA COMPLEJIDAD. UNA REFLEXIÓN SOBRE LA PERTINENCIA METODOLÓGICA EN CIENCIAS SOCIO-SANITARIAS Y HUMANAS
}

\author{
Siles González, J; Solano Ruiz, C; Cibanal Juán, L.
} Departamento de Enfermería. Universidad de Alicante.

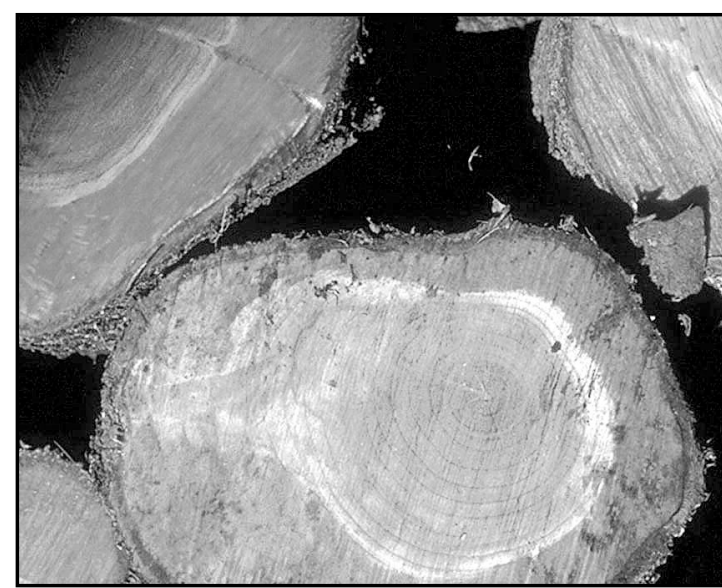

HOLISM AND QUALITATIVE RESEARCH WITHIN THE FRAMEWORK OF THE ANTHROPOLOGY OF COMPLEXITY. A REFLECTION ON THE METHODOLOGICAL PERTINENCE IN SOCIO-SANITARY AND HUMAN SCIENCES

\section{ABSTRACT}

This study was organised around the following objectives: Relating the principle of methodological and instrumental pertinence to the characteristics of the object-subject on which research is done within the epistemological framework of a discipline, and Reflecting on the pertinence of qualitative research and historico-ethnographic sources in socio-sanitary sciences (particularly nursing) and human sciences (due to their holism)

The theme development was structured through a process of analysis that focused on the three crucial phases in Edgar Morin's work: the Dialectics between the real and the imaginary (anthropology of death); the anthropocosmological dialectics, and finally, the anthropo-bio-cosmic dialogics.
Conclusions: From Morin on, the vision of reality became more multidimensional and holistic, since until well into the 20th century, knowledge had been based on abstraction, subdivision, simplification, reductionism, law generation, etc. Morin wanted to humanise science through a transdisciplinary anthropology in which the experiential dynamics of the human being was considered in a more global way starting from the paradigm of complexity, the qualitative methodology being a pertinent instrument for holistic clarification.

Keywords: Qualitative research, anthropology of education, anthropology of complexity, nursing.

\section{RESUMEN}

Este estudio se ha orientado partiendo de los siguientes objetivos: Vincular el principio de pertinencia metodológica e instrumental a las características del objeto-sujeto investigado en el marco epistemológico de una disciplina. Reflexionar sobre la pertinencia de la investigación cualitativa y las fuentes histórico-etnográficas en ciencias socio-sanitarias y humanas (particularmente la enfermería) y humanas (debido a su holismo)

El desarrollo del tema se ha vertebrado mediante un proceso de análisis centrado en las tres fases cruciales en la obra de Edgar Morin: Dialéctica entre lo real y lo imaginario (antropología de la muerte); dialéctica antropocosmológica y, por último desarrolla la dialógica antropo-bio-cósmica. Conclusiones: A partir de Morin se vislumbra una visión de la realidad más multidimensional y holística, dado que hasta bien entrado el siglo XX, el conocimiento se basaba en abstracción, parcelación, simplificación, reduccionismo, generación de leyes, etc. Morin pretende humanizar la ciencia mediante una antropología transdisciplinar en la 
que la dinámica experiencial del ser humano se contempla de forma más global partiendo del paradigma de la complejidad, constituyendo la metodología cualitativa un instrumento pertinente para la clarificación holística.

Palabras clave: Investigación cualitativa, antropología de la educación, antropología de la complejidad, enfermería.

\section{INTRODUCCIÓN}

Un fenómeno complejo ha sido definido como: " el compuesto por una gran variedad de elementos que mantienen entre sí una gran variedad de relaciones, con interacciones lineales y no lineales, sincrónicas y diacrónicas, la evolución de cuyo conjunto es imprevisible, incluso cuando su autoorganización se orienta por acciones teleológicas" (Vilar, 1997). Sin embargo, hoy parece evidente que el reduccionismo o la fragmentación de la realidad estudiada constituye un problema cuya relevancia no debe enmascararse por muchos que sean los impedimentos que obstaculizan su estudio sin renunciar a una perspectiva global de los fenómenos. Si la disciplina enfermera tiene por objetosujeto de estudio al hombre en el contexto de los cuidados de salud, se debe partir de un cuestionamiento previo a todo proceso de abordaje metodológico:

- ¿Qué tipo de conocimiento se pretende obtener... global o, por el contrario, se persigue un tipo de conocimiento parcial?

- ¿Se persigue la comprensión de la situación por parte del investigador ...es, por tanto precisa la comunicación?

- ¿El significado del proceso/ producto de la investigación es tan importante para el investigador como para el investigado?

\section{OBJETIVOS}

- Vincular el principio de pertinencia metodológica e instrumental a las características del objeto-sujeto investigado en el marco epistemológico de una disciplina.

- Explicar y clarificar los principios de la antropología de la complejidad y su relación con los paradigmas científicos y la enfermería.

- Identificar la pertinencia de la investigación cualitativa y las fuentes histórico-etnográficas en ciencias socio-sanitarias y humanas (particularmente la enfermería).

- Explicitar las relaciones entre la metodología narrativa, la dialéctica y reflexionar sobre su carácter dinámico.

\section{Ideas de partida}

- La realidad del ser humano es compleja: biológica, social, psicológica, política....

- Cuanto más reduccionista sea el método empleado para estudiar las situaciones vida.salud, más se estará mutilando la realidad observada.

- La investigación cualitativa permite obtener una visión holística, comprensiva y contextualizada de los fenómenos relacionados con los cuidados de salud.

- Metodológicamente holismo y dialéctica están vinculados por su carácter dinámico y direccional dado que ambos persiguen de una $u$ otra forma visiones sintéticas de los fenómenos.

- Las situaciones vida-salud-enfermedad-muerte no pueden ser interpretadas sin considerar su carácter procesual/dinámico.

- Los métodos y técnicas etnográficos e histórico-narrativos empleados en investigación cualitativa son los que mejor adaptan la complejidad al carácter procesual de la realidad estudiada desde una perspectiva holística.

\section{ESTADO DE LA CUESTIÓN}

a) Dinámica metodológica y realidad social cambiante:

La pertinencia metodológica como instrumento de indagación de la realidad social, ha sido cuestionada, fundamentalmente, en lo que se refiere a las ciencias sociales, filosóficas, históricas y antropológicas. Desde las aportaciones disímiles de la metodología dialéctica, la realidad ha sido objeto de estudio sin renunciar a su carácter dinámico: desde el revisionismo hegeliano (Kojeve, 1994, 2000), al materialismo dialéctico (Engels, 1978, 1980, 1990, 1997), se han planteado cuestiones sin las que sería imposible entender de autores que han evolucionado, como Edgar Morin, desde planteamientos fundamentados en la dialéctica. Ya han sido, pues, muchos los investigadores que han intentado superar las limitaciones metodológicas que no contemplaban la realidad de lo humano sin caer en reduccionismos exagerados. En esta línea 
hay que interpretar el fenómeno de la aparición de alternativas metodológicas más integradoras $\mathrm{u}$ holísticas. Sobre la antropología de la complejidad y el paradigma perdido (Morin, 1983a) existen gran número de trabajos. En esta misma línea de concienciación de la complejidad del fenómeno humano, pero enfatizando la dimensión biológica -reinterpretando lo biológico como factor primordial en la integración biopsicosocial- hay que reseñar las aportaciones de Maturana quien explicita la presencia de la dimensión biológica en todo lo humano: lenguaje (1978), la fundamentación biológica de la conciencia $(1987,1995)$.

Uno de los campos en los que más se han empleado los enfoques y usos dialécticos es en la psiquiatría y el contexto social de toda enfermedad: para comprender la relación dialéctica entre las conductas y las actitudes de los pacientes (Cunningham, Wolbert, Graciano; 2005); aplicando el método dialéctico a las dimensiones en las que anida el origen de la esquizofrenia: genética (tesis), epigenética (antítesis) y resolución final (síntesis) (tesis, antitesis, síntesis) para reflexionar sobre el origen de la esquizofrenia (Petronis, 2004), la dialéctica entre los fenómenos de inclusión-rechazo social inherentes a cierto tipo de enfermedades (Labonte, 2004); y, en fin, más genéricamente la dialéctica también se ha empleado para reflexionar entre dos supuestos antagonismos que parecen todavía difíciles de conciliar: el cientifismo y el humanismo (Ramey \& Chrysikou, 2005).

En la disciplina enfermera han sido varios los autores que se han ocupado de valorar la influencia del método dialéctico en el desarrollo de investigaciones de temática muy variada: el estudio y análisis de los arquetipos que se construyen socialmente partiendo de las creencias y valores asentados en la sociedad y que vertebran la dinámica de instituciones implicadas en la dispensación de cuidados de salud (familia, sistema sanitario y de ayuda social, etc.) (Kitchener \& Harrington,, 2004); Mohr (1999), desarrolla un estudio descriptivo y exploratorio implementando el método interpretativo e interaccionista de Denzin, para demostrar la naturaleza dialéctica de los cuidados.

b) Paradigmas científicos y pertinencia metodológica: el paradigma de la complejidad
La pertinencia metodológica y la relación entre la adopción de un tipo u otro de paradigma en el marco científico ha sido objeto de estudio por diversos autores en diferentes disciplinas: ciencias de la educación (López, 2000), historia (Aróstegui, 1999), liderazgo y economía (Arboledas,2001), arquitectura y cultura (Muntañola, 1999), medicina psiquiátrica (Lanteri-Laura, 2000) y hasta especialidades acotadas como el cáncer colo-rectal (Aranda, 2002) y otras muchas; sin embargo todas estas aportaciones relativas a diferentes disciplinas mantienen lo esencial de la adaptación realizada por Thomas Kuhn en su obra: "La estructura de las revoluciones científicas", dado que en todas las interpretaciones de paradigma prima la necesidad de clarificar modelos de identificación de problemas y soluciones, métodos y modelos adecuados y, por ende, la relación coherente de todo ello con sus respectivos marcos teóricos (Kuhn, 2000). Morin fue considerado como el pionero en el tema de la complejidad y lo hizo después de un período de reflexión que provocó cambios metodológicos y personales: el método se convirtió en una obsesión para él llegando a acuñar la expresión "el paradigma perdido" como concepto que reivindicaba la vuelta a una visión más global y humanista de la ciencia $(1981,1982,1983 b)$. Fruto de su tenacidad por adaptar de forma continua un método adecuado a la dinámica de la realidad social, tras un proceso de reflexión epistemológica (1990) llegó a proponer la superación de dicotomías tan tradicionales en la ciencia como la separación entre diferentes dimensiones: biológico, social, cultural; llegando a acuñar el concepto pensamiento complejo en diferentes obras $(1994,1996)$.

En España, son varios los autores que han contribuido a la difusión de este nuevo paradigma: Gómez ha estudiado la gran relación entre la dimensión experiencial de Morin y la evolución de su pensamiento (Gómez, 1996). Solana ha abordado la complejidad de la antropología incidiendo, asimismo, en el carácter tradicionalmente reduccionista de los métodos asentados en el marco de la ciencia (1999, 2001).

En el marco de la disciplina enfermera diversos autores han abordado el concepto de paradigma. Vinculado a la pertinencia epistemológica y metodológica como herramientas básicas de investigación (Siles, 1995, 1997a); sobre el tema de la 
coherencia entre métodos, modelos y paradigmas enfermeros, Botha (1989) realiza un trabajo aclaratorio sobre las analogías entre dominios incongruentes que suponen puntos de partida que, siendo erróneos, facilitan la superación de esa distorsión mediante la creatividad del hombre aplicándola a un lenguaje metafórico y teórico-génico. Otros autores mantienen que las diferencias entre investigación cualitativa y cuantitativa llega hasta los mismos cauces del paradigma en cuestión impregnando tanto las teorías como los modelos de caracteres diferenciales (Paley, 2000; Mitchell \& Pilkintong, 1999). Mención aparte merece el estudio sobre la incidencia del factor tiempo en el proceso de selección metodológico: cualitativo-cuantitativo, con sus respectivos métodos y técnicas; pero también afecta dicho factor temporal a la estructura de los datos recolectados y al análisis de los mismos (Sandelowski, 1999). Montu y Tingen (1999) repasan los diferentes paradigmas de forma crítica, revisando tanto el propio concepto de paradigma como los mimbres que mejor se adaptan a la naturaleza de la ciencia enfermera

c) La investigación cualitativa y los métodos histórico-etnográficos y narrativos como instrumento de superación del reduccionismo inmovilista de la ciencia y facilitador de una visión compleja de la realidad.

La dinámica de la realidad y los fenómenos emergentes que, de forma imprevista, se adhieren a la conformación de la misma, hacen que sea imposible estudiar los fenómenos humanos como si se tratara de una foto fija. La etnografía y la historia han sido pioneras en la utilización de materiales y métodos narrativos, siendo tantos los autores que han tratado el tema que su relación desbordaría el marco de este apartado; baste, pues, señalar dos que han contribuido al desarrollo de esa metodología: Pujadas, contribuyó a la utilización de la narrativa experiencial para el diseño y elaboración de historias y relatos de vida (Pujadas, 1992); Geertz ha llegado a comparar la actividad de un investigador social, como el antropólogo, a la actividad del autor (Geertz, 1989).La narrativa ha sido también muy utilizada en psicoanálsis, dado que el mismo Freud utilizó a los clásicos para desarrollar sus teorías (Yuman, 2001). En el marco de las ciencias de la salud, resultan destacables las aportacio- nes de Susan Sontag mediante sus trabajos sobre la vinculación del lenguaje y las enfermedades (Sontag, 1998) McIntyre describe las aportaciones de Sally Gadows a la enfermería mediante la utilización de materiales narrativos contando con mimbres como los pensamientos, perspectivas y experiencias para desentrañar la raíz de los significados que tienen las experiencias vida-salud-enfermedad. Otros autores han explicitado la validez de la narrativa como almacén experiencial en el que los fenómenos vida-salud se encuentran interrelacionados de forma holística (Siles, 1993, 1997b, 2000; Solano, 2003).Gadow aborda dialécticamente la relación cuerpo-narración en el contexto de la salud de la mujer (1994).

Otro de los fenómenos susceptibles de estudio mediante la metodología narrativa y etnográfica lo constituye la dinámica migratoria, cada vez más masiva, con toda la problemática que conlleva y cuyas coordenadas hunden sus raíces en la dialéctica enculturación-aculturación. (Bernard, 1987; Stockdale \& Warelow, 2000)

Otros autores han investigado la complejidad de los cuidados de salud como resultado de contradicciones y paradojas entrelazadas como desequilibrio o caos y también como orden no aparente considerando la enfermedad como un desorden difícil de reequilibrar en el sistema fisiológico del ser humano (Peña y Rodríguez, 2003); y, en fin, otros que han apostado por la extremada pertinencia de los métodos histórico etnográficos y la antropología de la complejidad en el marco de la enfermería

\section{MATERIAL Y MÉTODOS}

Al tratarse de un trabajo a medio camino entre la revisión, análisis y reinterpretación de los materiales desde una nueva perspectiva -diferente a la original de los mismos-, su vertebración responde a una necesidad de síntesis que intenta superar la tradicional división entre los catalogados como "originales" y "revisiones".

Se ha procedido, en primer lugar a realizar el proceso heurístico de búsqueda de estudios relacionados con el tema empleando las siguientes bases de datos: MEDLINE, CINAHL, CUIDEN y CUIDATGE.

Una vez extraídos los datos, se procedió al análisis de contenido y fueron agrupando en tres bloques de contenido temático por niveles de homo- 
geneidad: a) Dinámica metodológica y realidad social cambiante; b) Paradigmas científicos y pertinencia metodológica; c) La investigación cualitativa y los métodos histórico-etnográficos y narrativos como instrumento de superación del reduccionismo inmovilista de la ciencia y facilitador de una visión compleja de la realidad.

Posteriormente se procedió a analizar comparativamente las características de la antropología de la complejidad y la disciplina enfermera con la finalidad específica de identificar las semejanzas y diferencias epistemológicas y metodológicas entre ambas.

\section{DESARROLLO DEL TEMA}

A. Dinámica metodológica y realidad social cambiante

a) La salud y la enfermedad como un proceso situacional.

En la ciencia se han ido sucediendo diferentes modelos para interpretar el principio de pertinencia metodológica e instrumental a las características del objeto-sujeto investigado en el marco epistemológico de una disciplina. El primer método que respeta el carácter dinámico de la realidad integrando en una síntesis procesual las diferentes partes que la integran, es, el método dialéctico hegeliano reinterpretado por Kojeve $(1994,2000)$ y el materialismo dialéctico e histórico desarrollado por Marx (1993) y Engels (1978, 1990, 1997). Siguiendo a este último autor se puede constatar el carácter dinámico de la realidad:

"La dialéctica no es más que la ciencia de las leyes generales del movimiento y la evolución de la naturaleza, de la sociedad humana y del pensamiento." (Engels, 1978)

En este contexto iluminado por el razonamiento dialéctico, los conceptos de salud y enfermedad deberían compartir el carácter dinámico o procesual:

“(...) Salud es el ajuste perfecto y continuo del hombre a su ambiente; por el contrario la enfermedad es un ajuste imperfecto y discontinuo (...)" (Domínguez, 1988)

Por otro lado, la dimensión situacional y dinámica de todo lo que tiene que ver con la salud y la enfermedad se expresa en la siguiente expresión:

"No existen la salud y la enfermedad (como expresiones universales o absolutas), sino, simple- mente, la suma de interpretaciones derivadas del conjunto de situaciones implicadas en un proceso" (Siles, 2000)

La dialéctica, como instrumento de indagación, razonamiento e interpretación de la realidad ha sido interpretada de forma diferente según la época histórica.

Heraclito (500 a.C) afirmaba que: "todas las cosas son y no son, porque todo fluye, está cambiando constantemente, constantemente naciendo y muriendo. Es imposible sumergirse dos veces en uno e idéntico rió (...) La misma cosa en nosotros vive y muere, duerme y está despierta, es joven y vieja; cada una cambia su lugar y deviene la otra. Nosotros entramos y no entramos en el mismo rió: estamos y no estamos “.

Esta es la esencia del método dialéctico, el cambio continuo a través del tiempo y mediante la sucesión de contradicciones que se resuelven en síntesis continuadas.

Engels, en su obra dedicada a Ludivlg Feuerbach y el fin de la filosofía clásica alemana, define la dialéctica de forma dinámica y enfatiza el carácter procesual y el principio de homeostasis que mantiene un cierto equilibrio en los cambios que se producen:

"La gran idea cardinal de que el mundo no puede concebirse como un conjunto de objetos terminados y acabados, sino como un conjunto de procesos, en el que la co-sas que parecen estables, al igual que sus reflejos mentales en nuestras cabezas, los conceptos, pasan por una serie ininterrumpida de cambios, por un proceso de géne-sis $\mathrm{y}$ caducidad (...) Para la filosofía dialéctica no existe nada definitivo, absoluto, consagrado; en todo pone de relieve lo que tiene de perecedero, y no deja en pie más que el proceso ininterrumpido del de-venir y perecer, un ascenso sin fin de lo inferior a lo superior, cuyo mero reflejo en el cerebro pensante es esta misma filosofía." (Engels, 1997)

El error de interpretar cualquiera de los momentos en los que se encuentra el proceso: salud-vida-enfermedad de forma estática (foto fija), se deriva de planteamientos anclados en una interpretación metafísica del fenómeno, para el metafísico sólo existe enfermedad como realidad aislada y en confrontación con otra realidad igualmente confinada: la salud.

"Para el metafísico las cosas y sus imágenes en 
el pensamiento, los conceptos, son objetos dados de una vez para siempre, aislados, uno tras otro y sin necesidad de contemplar el otro, firmes, fijos y rígidos (...) Para él, toda cosa existe o no existe: una cosa no puede ser al mismo tiempo ella misma y algo diferente. Lo positivo y lo negativo se excluyen lo uno a lo otro de un modo absoluto; la causa y el efecto se encuentran del mismo modo en rígida contraposición" (Engels, 1978).

b) Dos conceptos nucleares para la comprensión de los cuidados de salud y la naturaleza de la investigación cualitativa: homeostasis y holismo como instrumentos para interpretar dinámica y globalmente las situaciones: vida-salud-enfermedad

*Homeostasis y el paradigma tetralógico de Morín (orden/desorden/interacciones/organización).

Se puede interpretar la homeostasis, en principio, como algo que opone resistencia al cambio como: la tendencia a la estabilización del cuerpo relacionado con los procesos fisiológicos que están continuamente interactuando, provocando cambios continuos que pasan desapercibidos en aras al mantenimiento de una manifiesta estabilidad de orden superior. El medio interno responde a los cambios del medio externo que rodea al organismo. Se podría interpretar la homeostasis como una propiedad que controla su nivel de respuesta y adapación al contexto $y$, en ese sentido, es un factor sistémico que facilita el equilibrio y la estabilidad del mismo (Maturana, 1972).

La homeostasis social se puede estudiar observando, por ejemplo, la resistencia que las estructuras oponen a los cambios históricos. La familia, como estructura social básica de convivencia y socialización (Siles, 1999), actúa como un mecanismo interno de estabilización de la sociedad, pero cuando se producen cambios radicales en la sociedad, la familia también suele evolucionar de forma convulsa. En definitiva se podría afirmar que la homeostasis responde a una dialéctica en la que intervienen los elementos de sistemas internos y externos y en los que el resultado es un proceso dinámico de síntesis.

En los procesos dialécticos integrados en las situaciones de salud-enfermedad, se producen continuamente cambios e interacciones que determi- nan el predominio de un tipo u otro de evolución según prevalezca una tendencia hacia el mantenimiento de la salud (ajuste perfecto y continuo del hombre a su ambiente...), o, por el contrario el rumbo se oriente hacia una enfermedad (ajuste imperfecto y discontinuo).

El mecanismo regulador o autoorganizador de la homeostasis tiene cierta equivalencia conlos principios del paradigma tetralógico: orden/desorden/interacciones/organización. Este paradigma es igualmente válido para la dimensión biológica y para la antropológica (Morin 1980). La autoorganización se produce mediante los genes y su relación con el medio: el fenotipo. El fenotipo, también está sujeto a una dinámica reguladora semejante en lo esencial a la homeostasis, es una característica observable, identificable e individualizada del organismo, que expresa un genotipo específico en un ambiente determinado de forma que existen dos clases de fenotipos: el fenotipo potencial y el fenotipo real. El fenotipo potencial de una persona es el que se desarrollaría si sólo si alguien llegara a estar en las condiciones ambientales proclives a tal hecho, mientras que el fenotipo real es el que expresa al individuo como producto de su interacción con el medio (Fenotipo real $=$ genotipo + ambiente)

* La perspectiva holística como instrumentos para interpretar dinámica y globalmente las situaciones: vida-salud-enfermedad

El holismo, como forma de interpretar la realidad, constituye un enfoque global cuya finalidad radica en superar los límites de los compartimen-

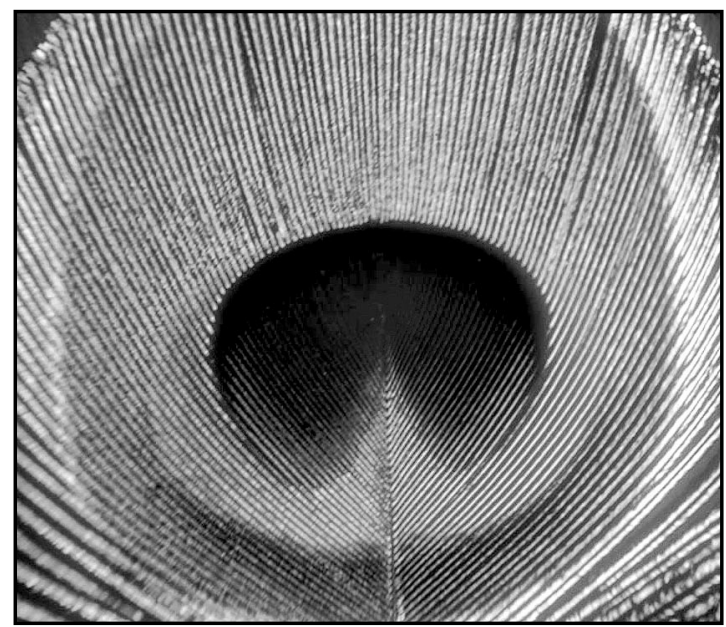


tos estancos que constituyen los lindes de la investigación cuantitativa. Uno de los apoyos más sólidos de la visión holística de los fenómenos -tal como señala Prigogine (1991), radica en la perspectiva histórica, dado que todas las variaciones que se producen en cualquier sistema lo hacen a través del tiempo: "no se puede comprender un sistema complejo más que refiriéndose a su historia y su transcurso".

El cambio como principio dialéctico que dinamiza la realidad, en todos sus aspectos, precipita la obsolescencia de los estudios que se centran en etapas concretas y aisladas de los fenómenos aportando resultados tan fragmentarios como efímeros. Es por ello que algunos autores han intentado demostrar la importancia de la adopción de métodos cuya flexibilidad permite amoldar la necesidad de conocimiento de un tramo o momento del proceso con la provisionalidad del mismo previniendo la sucesión de cambios inminentes. Es el caso de Crocker que aporta el paradigma funcional como instrumento metodológico cuya apertura y flexibilidad permite la integración de necesidades educativas y soluciones emergentes en el transcurso de la práctica educativa erigida en el núcleo de una investigación procesual (Crocker, 1986). En este mismo sentido parecen encaminarse las aportaciones de autores que postulan la investigación enfermera centrada en la práctica de los cuidados (Malvárez, 2002; Hesook, 1993). El paradigma funcional, implementado en enfermería, supondría un intento de captar todas las circunstancias, elementos y factores emergentes (siempre potenciales), que determinan un momento dentro de amplio y variado proceso salud-enfermedad, lo cual implica aplicar la observación, el análisis y la interpretación en cada uno de esos momentos en los que se realiza la práctica de los cuidados.

Desde el punto de vista de los cuidados de enfermería, el paradigma de la complejidad favorece una visión global y holística del individuo, evitando la relación lineal de causa efecto, el reduccionismo y la fragmentación y contemplando la influencia de factores psicosociales y culturales que pueden favorecer los desordenes.

B. Paradigmas científicos y pertinencia metodológica
El término paradigma fue acuñado por Kuhn adaptándolo al contexto científico en el que le confirió distintos significados: paradigma como grupo de científicos que han consensuado diferentes formas de lenguaje, identificación y resolución de problemas; como colegio invisible o sistema de citas mutuas, y, por último, lo definió como: "un sistema de referencias que sirven para aglutinar los ejemplares compartidos que caracterizan una rama de la ciencia" (Kuhn, 2000, Siles, 1996). En definitiva un paradigma constituye un soporte que sirve para que la ciencia se desarrolle cooperativamente integrando los diferentes grupos de investigadores mediante un proceso de socialización específicamente científico. Según el tipo de paradigma el mismo fenómeno tiene diferentes temas de interpretación. A lo largo de la historia, esencialmente, se han sucedido cuatro grandes paradigmas: teológico (interpretación sobrenatural de los fenómenos, particularmente los relacionados con la muerte y los desastres naturales y la enfermedad) (Tabla I), racional tecnológico o neopositivista (interpretación racional y objetivada de la realidad) (Tabla II), hermenéutico (en cuyo marco los fenómenos son susceptibles de subjetivación, individualización y comunicación (Tabla III) y, por último, el sociocrítico (Tabla IV) (la interpretación considera los mismos factores contextuales que el anterior pero, además, constituye la primera fase para la acción transformadora de la realidad) (Siles y García, 1995; Siles, 1997b)

\section{* Edgar Morin, la superación del dogma y la Antropología de la Complejidad}

El origen de la evolución de Morin hacia la antropología de la complejidad hay que buscarlo, en una serie de situaciones personales y circunstanciales:

- Crisis del dogma marxista

- Ingreso en el Centro Nacional de Investigaciones Científicas (1950)

- Necesidad de superar la ortopedia dogmática del marxismo.

- Evolución entre el azar y la estrategia (intuición).

Gómez (1996) destaca tres fases en la evolución de Morín hacia la antropología de la complejidad: un nuevo paradigma 


\section{Antropología de la muerte: lo real y lo ima- ginario}

En la primera etapa ingresa en el CNRS y publicación de: "El hombre y la muerte" (1951) y "El cine o el hombre imaginario" (1956). Revisa el método marxista, pero intenta salvarlo mediante una ampliación de su dialéctica que permita la visión de los planos biológico-físico y lo imaginario (persigue la visión del hombre total).El texto de referencia en esta época es todavía marxista: Manuscrito económico fiolosófico. Morin confiesa que de él extrae la idea de hombre genérico y pergeña el objetivo de alcanzar el hombre total. En el manuscrito argüía que la ciencia del hombre y la ciencia de la naturaleza debían mantener una relación dialéctica sin llegar nunca a engullirse mutuamente. Asimismo le influyó la idea de Marx en la que unía teoría y praxis ene una misma concepción (Morin, 1994). Se encuentra embuído, en este período, en una epistemología dialéctica que instrumentaliza para estudiar las dialécticas del hombre y la naturaleza. Morin considera normales e incluso necesarias situaciones de regresión, inadaptación y alineación; dado que todas, globalmente, hacen posible la evolución.

En este sentido la enfermedad y la salud constituyen situaciones normales en el curso de una relación dialéctica que, de forma sucesiva, se resuelve en síntesis.

En su obra "El hombre y la muerte" (1951) desarrolla una antropología de la muerte vertebrada en una triple constante antropológica:

- Conciencia de ruptura que conlleva la muerte.

- El daño o traumatismo que esta conciencia/ saber inflinge.

- La aspiración a la inmortalidad.

La realidad científica no es del todo real, al menos...no en su totalidad. Mediante la imaginación y la creatividad, el hombre ha intentado interpretar la realidad de forma que pudiera entenderla $\mathrm{y}$, si era posible, darle un sentido...un significado. La enfermedad, el dolor, las catástrofes, la muerte. En el paradigma teológico se predicaba la verdad absoluta mediante la solución totalitaria que ofrecían las religiones. En definitiva, hasta hace poco, la ciencia se mantenía al margen de las visiones globales u holísticas de la realidad, sin considerar el principio de que: "El todo es inexplicable sin referirlo a las partes, del mismo modo que las par- tes son incompresibles sin relacionarlas en el todo" (Vilar, 1997). Morin une la realidad y el producto creativo de la imaginación del hombre (mitos, religiones, etc.), dado que lo imaginario cumple una función plenamente real, dado que no existen dos fuentes del devenir humano, una racional y otra mítica, sino que el mismo movimento produce útiles y mitos que se adaptan al mundo biológico y aun lo sobrepasan (Morin, 1959). El hombre utiliza la creatividad como instrumento para superar las contradicciones y frustraciones que provoca la muerte finiquitando su individualidad:

- Elaboración de concepciones de la muerte en un contexto bipolar:

a) Cosmomorfismo: inspirado en el recurrente renacimiento de la vida en la naturaleza (muerteresurrección o muerte-descanso eterno: ritos, religiones, celebraciones de finales y comienzos de ciclos).

b) Antropomorfismo: mantiene la individualidad mediante la vía de la innmortalidad: individuo amortal. Esta condición amortal se logra mediante un doble camino:

- Mediante la creatividad que produce un imaginario doble del individuo (alma, superhéroe, etc), que le preserva de la muerte/ desaparición.

- Por medio de la ciencia y la tecnología (genóma como paradoja: la parte está en el todo, pero el todo también se encuentra en la parte)

\section{Crisis teórica, política y personal (enferme- dad)}

En esta segunda fase ,marcada por una doble crisis (de salud y de dogma), escribe un diario que va a dar lugar a dos publicaciones: "Introducción a una política del hombre" (1965) y "Lo vivo del sujeto" (1969). En este período su objetivo se va a ir perfilando al ritmo de sus reflexiones y la progresiva recuperación de su deteriorada salud. Morin se propone, una vez desengañado por las limitaciones del materialismo dialéctico, superar la dialéctica de la totalidad mediante su proyecto del hombre inserto en el mundo (antropocosmología). La diversidad de sus fuentes es cada vez mayor (relatividad de Einstein, principio de incertidumbre de Heinseberg, la antimateria, cibernética, la teoría 


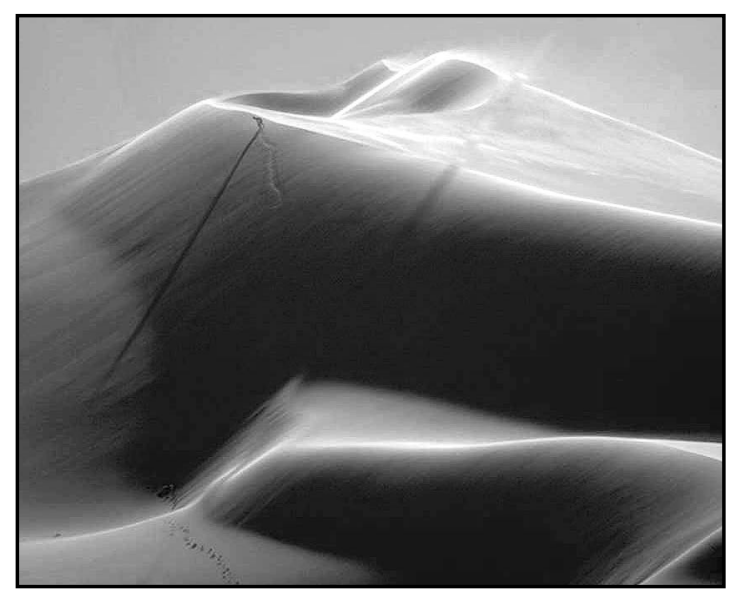

de la información, etc.)y la heterogeneidad la asimila de forma sintética manteniendo como constante una progresiva globalidad en su visión del hombre y la naturaleza.

Se enfrasca la elaboración de una visión antropocosmológica del hombre en la que hombre y mundo están coimplicados y la fundamenta en la naturaleza caótica del mundo y en la condición "histérica" del hombre.

- Metodológicamente retiene puntos clave de disímiles procedencias metodológicas:

* Análisis de los períodos históricos estructurados (Marx).

* Elucidación de fases afectivas estructuradas (Freud).

* Idea sobre una antropogenética (Piaget).

Propone una teoría multidimensional y la denomina: "Radicales Antropológicos o antropocosmológicos" que responden, igualmente, a un eclecticismo metodológico caca vez más radical:.

* Producción (Marx)

* Psique (Freud)

* El amor (cristianismo)

* La ciencia moderna

* La poesía (reivindicada por el superrealismo)

Pero en esta segunda fase no alcanza a construir un método, se queda en las ideas.

3. El origen de un nuevo paradigma: la antropología de la complejidad.

A partir de los cincuenta años, Morin entra en fase de madurez coincidiendo con su ingreso en el El instituto Salk para ciencias biológicas
(California), publicando su "Diario de California" (1970), "El Paradigma perdido: la naturaleza humana"(1973) y en sucesivas etapas"El método $(1,1977 ; 2,1980 ; 3,1986 ; 4,1991)$ ". En esta nueva etapa se vuelve a reformular sus objetivos e impulsado por conocimientos de la nueva biología empieza a desarrollar la antropología de la complejidad intentando potenciar la visión holística y, a la vez, dialéctica: vida- muerte, biológico-filosófico, etc.En esta etapa californiana elabora su antropología de la complejidad que se cimenta en tres pies (articulación entre physis, bios y ánthropos):

- Antropocosmología

- Antropobiología

- Antroposociología

C. La investigación cualitativa y los métodos histórico-etnográficos y narrativos como instrumento de superación del reduccionismo inmovilista de la ciencia y facilitador de una visión compleja de la realidad.

- Planteamientos previos o reflexiones en torno a la adopción metodológica.

Por un lado la investigación ha sido definida como el proceso sistemático de indagación en el conocimiento de una disciplina mediante la aplicación de métodos y técnicas adecuados; por otro la investigación cualitativa se puede entender como: la investigación de un fenómeno particular realizada en profundidad y de una forma holística a través de la recogida de información narrativa valiosa y usando un diseño de investigación flexible (Polit \& Hungler, 1997)La realidad se compone de factores de naturaleza diversa que se combinan de forma dinámica y múltiple dando lugar a visiones superficiales del fenómeno que comporta y explicitando un orden a menudo sólo aparente. Las enfermedades y los cuidados de salud constituyen una parcela de la realidad en la que funciona el mismo mecanismo con idéntico, o mayor si cabe, nivel de complejidad interrelacional. Si el objetivo de cualquier investigación no conlleva superar las apariencias que distorsionan la casuística radical del fenómeno objeto de estudio, entonces no será necesario nada de esto, pero será evidente que el trabajo realizado carece de visión holística y que 
sus resultados caerán en la parcialidad más palmaria. $\mathrm{Si}$, por el contrario, entre nuestros objetivos, se encuentra desarrollar una perspectiva global del fenómeno y, por ende, se pretende alcanzar la comprensión del mismo llegando a la raíz de su casuística, se deberán tomar todas las medidas de "profilaxis metodológica" reseñadas anteriormente.

Las rutinas procedimentales y las técnicas estandarizadas deberán dejar paso a un estudio etnográfico-histórico-narrativo del objeto sujeto investigado, pero también del contexto en el que se encuentra, dado que desde el paradigma de la complejidad, el individuo y el medio ambiente interactúan y se parte, además de conceptos dinámicos e interrelacionales de la salud y la enfermedad (Domínguez, 1988).

La investigación centrada en la práctica de los cuidados, convenientemente reinterpretada a la luz del paradigma de la complejidad, puede ser un escenario adecuado mediante la realización un esfuerzo teórico adicional que vaya más allá de la mera aplicación rutinaria de métodos y técnicas teniendo en cuenta el contexto, dado que habría que adaptar continuamente la herramienta indagatoria a la dinámica contextual (de ahí la importancia de la flexibilidad y vigilancia constantes de las características del entorno y del propio objetosujeto investigado) (Crocker, 1986; Malvárez, 2002; Hesook, 1993). Romin (2003), estudia las implicaciones de la narrativa en las vivencias y en la práctica profesional y educativa de enfermería, llegando a proponer un modelo dialéctico. vertebrado en tres fases o aproximaciones éticas: inmersión subjetiva (contexto ético), separación objetiva (ética universal), y compromiso intersubjetivo (narrativa relacional). Gadows (1994) desarrolla un proceso dialéctico entre el cuerpo y la narrativa (¿De quién es este cuerpo, de quién es esta historia?) instrumentalizando esta relación en el contexto de los cuidados de salud de las mujeres.

- El flujo de conciencia como visualización de la relación entre la vida exterior y la vida interior: fenomenología y cuidados de salud

Sobre aspectos específicos que se derivan de la utilización de métodos narrativos (escritos: diario de campo, historias y relatos de vida, entrevistas en profundidad, semiestructuradas, o audiovisuales: video), destaca el denominado "flujo de conciencia", o lo que es lo mismo los trabajos realizados sobre la relación entre la evolución personal de los pacientesy el desarrollo de sus situaciones de salud. Se puede considerar el "feed back", como una forma particular, muy limitada en el tiempo, de flujo de conciencia Siles,2000). Algunos autores sostienen un punto de vista biologicista del flujo de conciencia considerando al conocimiento e incluso al lenguaje como un fenómeno muy vinculado al plano biológico (Maturana, 1978, 1987). Sin embargo, otros trabajos abordan el tema de la complejidad humana desde una perspectiva antropológica mucho más amplia recomendando el empleo de métodos audiovisuales (Siles et al, $\left.2004^{\mathrm{a}}, 2004 \mathrm{~b}\right)$. El flujo de conciencia ha sido utilizado por los pioneros de la teoría de la complejidad para explicar la multidimensionalidad y la interrelación del gran número de factores que determinan un momento dado sin que, aparentemente, estos factores fundamentalmente experienciales, estén presentes. La narrativa se ha utilizado reiteradamente para describir la realidad sin renunciar a la complejidad que la conforma y teniendo en cuenta la influencia que las experiencias pretéritas de los sujetos-personajes tienen en un momento dado de sus vidas (Faulkner, Joyce, García Márquez, Wolf), o lo que viene a ser lo mismo, para identificar la influencia que la vida interior tiene en la vida exterior: el equilibrio homeostático correspondiente a la interrelación entre el plano interior y el exterior. La interrelación entre el desarrollo de la teoría de la complejidad y la literatura (más concretamente los usos literarios del denominado "flujo de conciencia" se deja translucir claramente en las palabras del escritor mejicano Gustavo Sainz:

“(...)Cada vez que escribo algo nuevo advierto que me cuesta más trabajo. Debería ser al revés, si creemos en la experiencia, pero la experiencia aquí no cuenta. Lo difícil, lo verdaderamente difícil es poder mirarse uno mismo, en todos sus dobleces y eventualidades, en todas sus metáforas, metamorfosis y proyecciones. En este libro propongo que el sujeto es lo menos estable que hay, siempre está en movimiento, en permanente alteración, planeando su siguiente movida, inquieto, en ebullición. Yo no soy ahora el mismo que seré mañana. El problema es que pese a que uso el 
mismo nombre y aparezco más o menos con el mismo físico, podrías decir que me conoces, pero nadie conoce a nadie. Digámoslo de otra manera: me puedes "interpretar", pero no conocer, y lo más seguro es que quizás me "malinterpretes". Con qué astucia decía Freud que empezaba a sospechar que en cada acto sexual intervenían por lo menos cuatro personas(...)" (Posadas, 2001)

Lo cierto es que la realidad llega a las personas a través de los sentidos mediante la observación que activa la interacción entre el plano biológico, dada la trascendente función canalizadora de los sentidos como mediadores entre el mundo externo percibido y el sujeto que interpreta los fenómenos, y la dimensión cultural-simbólica que impregna la actividad hermenéutica y comprensiva del hombre confiriéndole un gradiente que rebasa lo puramente biológico.

Tal como señala Merleau Ponty;:

- "Yo no soy el resultado o entrecruzamiento de las múltiples causalidades que determinan mi cuerpo o mi psiquismo, no puedo pensarme como una parte del mundo, como un simple objeto de la psicología y de la sociología, ni cerrar sobre mí el universo de la ciencia(...)" (Merleau-Ponty, 1988)

En consecuencia la fenomenología de la percepción puede, también, contribuir a una mayor amplitud de la perspectiva con la que se abordan los fenómenos vida-salud-enfermedad-muerte, pero en definitiva, siguiendo a Morin y sus radicales antropológicos (sistema de producción de Marx, psique de Freud, amor del cristianismo, ciencia moderna y poesía surrealista) cuanto más potencial de síntesis contengan los métodos utilizados mayor será la capacidad del investigador para eludir el riesgo de reduccionismo o mutilación del fenómeno investigado.

\section{DISCUSIÓN DE RESULTADOS}

- El concepto de paradigma ha sido utilizado de forma muy diversa según la interpretación de autores pertenecientes a diferentes tendencias y disciplinas. En este trabajo se han considerado los paradigmas históricos (teológico, racional tecnológico y sociocrítico), como los más extendidos en el tiempo y en su difusión. El paradigma de la complejidad responde a nuevas necesidades del hombre y también científicas, pero su evolución es aún una cuestión pendiente y su aplicabilidad resulta extremadamente complicada.

- Las personas ante la visión de los mismos fenómenos, aún bebiendo de las mismas fuentes dialécticas, compartiendo el tiempo histórico y haciendo frente a las mismas adversidades, no interpretan del mismo modo los acontecimientos. Autores tan reputados como Morin, Merleau Ponty y Sartre inician su andadura desde una fuente común: el materialismo dialéctico, a partir de ahí evolucionan personalmente de forma diversa (compromisos históricos frente a la invasión nazi de Francia) y llegan a vertebrar sus respectivas obras como producto de una diversidad hermenéutica: antropología de la complejidad, fenomenología de la percepción y existencialismo humanista.

- Algunos autores suelen usar las categorizaciones metafísicas de realidades como: salud, enfermedad, dolor, etc.; que se contraponen a conceptualizaciones dialécticas de las mismas realidades, las primeras comportan significados que denotan realidades estáticas, mientras que las interpretaciones dialécticas de situaciones de salud, enfermedad, dolor, etc., implican la visiones dinámicas y procesuales de dichos fenómenos. Siendo necesario que lo eidético o esencial de los fenómenos tenga un carácter universal y que el reduccionismo fenomenológico-epistemológico socave el dinamismo de los conceptos mencionados vaciando sus significados de las variantes contextuales e históricas, dado que tal como señala Heiddeger para reclamar lo que significa la salud "real" (en un contexto y en un tiempo) se tiene que saber que saber ya previamente que significa la salud "en general" (Heidegger, 2000); se hace necesario, asimismo, clarificar en cada momento la perspectiva y el lenguaje con los que se abordan los fenómenos implicados en las situaciones vida-saludenfermedad .

- La utilización de la investigación cualitativa implica el estudio de las experiencias vida-saludenfermedad en la vida cotidiana, conjunto de fenómenos que constituyen un proceso en continua evolución y dialéctico, por lo que sus componentes contextuales e históricos deben integrarse tanto en su conceptualización como en el lenguaje empleado. 


\section{CONCLUSIONES}

- Considerando que la realidad del ser humano es compleja: biológica, social, psicológica y política; su dinámica experiencial se contempla de forma más global partiendo del paradigma de la complejidad.

- La antropología transdisciplinar aportada por Morin persigue la humanización de la ciencia ampliando su arsenal metodológico y epistemológico.

- La antropología de la complejidad tiene por objetivo resolver los enfoques fragmentarios y reduccionistas de la ciencia, mediante una síntesis metodológica, epistemológica y bilógica.

- Los métodos tradicionalmente empleados en la ciencia experimental resultan altamente reduccionistas a la hora de interpretar las realidades implicadas en situaciones vida.salud..

- La investigación cualitativa permite obtener una visión holística, comprensiva y contextualizada de los fenómenos relacionados con los cuidados de salud.

- El carácter procesual de los métodos holísticos y dialécticos permite un enfoque dinámico de las situaciones vida-salud-enfermedad.

- La etnografia y los métodos histórico-narrativos empleados en investigación cualitativa resultan idóneos para estudiar los fenómenos complejos que inciden en las situaciones vida-salud-enfermedad.

\section{BIBLIOGRAFIA}

- Aranda, E. (2002) Nuevos paradigmas en cáncer colorectal. You \& Us, Madrid.

- Arauzo, A.C; Watson, M;\& Hulgus, J. (1994) The clinical uses of video therapy in the treatment of childhood sexual trauma survivors. Journal of Chile Sexual Abuse, 3-4:37- 45

- Arboledas,J.R. (2001) Paradigmas del liderazgo. McGrawHill-Interamericana, Madrid.

- Bernard (1987) A dialectic of migration: acculturation and demographic transition.Eur J Popul..2(3-4):387-405

- Botha, M.E. (1989) Theory development in perspective: the role of conceptual frameworks and models in theory development. J Adv Nurs. 1989 Jan;14(1):49-55.

- Bruner, J. (1991). Actos de significado. Más allá de la revolución cognitiva. Madrid: Alianza.

- Coppa, D. (1993) Chaos Theory suggest a new paradigm for nursing science. Journal of Advance Nursing, 18: 985-991.

- Cornock, M. (1998) Stress and intensive care patient: perceptions of patients and nurses. Journal Advances Nurses, 27: 518-527.

- Cortés, J.M. (1997). Orden y Caos. Barcelona. Anagrama

- Crocker, C. (1986) El paradigma funcional de los profesores. Revista de Innovación e Investigación Educativa. 1:53-64.
- Cunningham K, Wolbert R, Graziano A, Slocum J.(2005) Acceptance and change: the dialectic of recovery. Psychiatr Rehabil J. 2005 Fall;29(2):146-8

- Da Silva, A.L. \& Ciampone, M.H. (2003) A paradigmatic glance on nursing care -- a walk to complex care.Rev Esc Enferm USP.;37(4):13-23.

- Grant, B.M. \& Giddins, L.S. (2002)Making sense of methodologies: a paradigm framework for the novice researcher. Contemp Nurse. 13(1):10-28

- Jotterand, F. (2005) The Hippocratic oath and contemporary medicine: dialectic between past ideals and present reality? J Med Philos.;30(1):107-28.

- Derrida, J. (1989) La escritura y la diferencia. Anthropos, Barcelona.

- Derrida, J. (1998) De la gramatología. Siglo XXI, México.

- Domínguez, M. (1988) Concepto de salud y enfermedad. En: Medicina preventiva y salud pública. Salvat, Barcelona.

- Engels, F. (1978) Anti-Dèhring. Ayuso, Madrid.

- Engels, F. (1980) El origen de la familia, de la propiedad privada y del Estado: en relación con las investigaciones de L.H. Morgan. Ayuso, Madrid.Engels, F. (1990) Introducción a la dialéctica de la naturaleza.Ediciones Vosa, Madrid.

- Engels, F. (1997) Ludwig Feverbach y el fin de la filosofía clásica alemana . Debarris, Barcelona

- Gadows, S. (1994) Whose body? Whose story? The question about narrative in women'shealth care.Sundings. FallWinter;77(3-4):295-307. No abstract available.

- Geertz, C. (1989) El antropólogo como autor. Paidós, Barcelona

- Gómez, P. (1996) Construcción de la antropología compleja de Edgar Morin. Gazeta de Antropología, 12, octubre: 11-18.

- Habermas, J. (1991) Teoría de la acción comunicativa. Taurus, Madrid.

- Heidegger, M. (2000) De la esencia de la verdad. Alianza, Madrid.

- Heidegger, M. El ser y el tiempo. Fondo de Cultura Económica, Madrid.

- Hesook, S.K. (1993). Putting theory into practice: Problems and prospects. Journal of Advanced Nursing, 18, 1632-1639.

- Kitchener, A.\& Harrington, C. (2004) The U.S. long-term care field: a dialectic analysis of institution dynamics.J Health Soc Behav. 2004;45:87-101.

- ${ }^{\circ}$ Kojeve, A. (1994) La idea de la muerte en Hegel. Leviatán, Buenos Aires.

- Kojève, A.(2000) Outline of a Phenomenology of Right. Rowman \& Littlefield, London.

- Kuhn, T. (2000) La estructura de las revoluciones científicas. Fondo de Cultura Económica, Madrid.

- Labonte, R (2004) Social inclusion/exclusion: dancing the dialectic. Health Promot Int.;19(1):115-21

- Lanteri-Laura, G. (2000) Ensayo sobre los paradigmas de la psiquiatría moderna. Triacastela, Guipúzcoa.

- López, J.A. (2000) Paradigmas y métodos pedagógicos para la educación social: La praxis pedagógica en educación social. Nau Libres, Valencia.

- Malvárez, S. (2002) Contexto de la practica centrada en los cuidados. Multideterminación, crisis y complejidad. Enfermería Global, 1: 2-5.

- Marx, K. (1993) Manuscritos: economía y filosofía. Altaya, Barcelona.

- Maturana, H. R. (1978), Biology of Language: The 
Epistemology of Reality" en G. A. Maturana, H. R. (1987), The Biological Foundation of Self Consciousness and the Physical Domain of Existence", en E. R. Caianiello (ed.), Physics of Cognitive Processes, Singapur, World Scientific, pp. 324-379.

- Maturana, H. R. (1990), "Science and Daily Life: The Ontology of Scientific Explanation", en W. Krohn, G. Kuppers y H. Nowotny (eds.), Selforganization: Portrait of a Scientific Revolution, Dodrecht, Kluwer Academic Publishers,. 12-35.

- Maturana, H. R. (1995), "Biology of Self Consciousness", en G. Tranteur (ed.), Consciousness: Distinction and Reflection, Nápoles, Bibliopolis.

- Maturana, H. R. y J. Mpodozis (1992), "Origen de las especies por medio de la deriva natural", Publicación ocasional, núm. 46, 1992, Santiago de Chile, Museo Natural de Historia Natural.

- Maturana, H., Varela, F.: De Máquinas y Seres Vivos, Chile, 1972, Ed. Universitaria.

- McIntyre, M. (2003) Cultivating a worldly repose: the contribution of Sally Gadow's work to interpretive inquiry. Nurs Philos. Jul;4(2):111-20.

- Merleau Pony, M. (1988). Fenomenología de la percepción. México: Fondo de Cultura Económica.

- Mitchell, G.J. Pilkintong, F.B. (1999) A dialogue on the comparability of research paradigms--and other theoretical things. Nurs Sci Q.;12(4):283-9.

- Montu, E.J. \& Tingen, N.S.(1999) Multiple paradigms of nursing science. ANS Adv NsCi21(4):64-80

- Paley, J. (2000) Paradigms and presuppositions: the difference between qualitative and quantitative research. Sch Inq Nurs Pract. 2000 Summer;14(2):143-55; discussion 157-63.

- Morin, E. (1983a) El paradigma perdido. Ensayo de bioantropología, Kairós, Barcelona

- Morin, E. (1981) El método, I: La naturaleza de la naturaleza, Madrid, Cátedra.

- Morin, E. (1983b) El método, II: La vida de la vida, Madrid, Cátedra.

- Morin, E. y Piattelli-Palmarini, M. (1983) La unidad del hombre como fundamento y aproximación interdisciplinaria, en: Interdisciplinariedad y ciencias humanas. Tecnos/UNESCO, Madrid: 188-212.

- Morin, E. (1994) Introducción al pensamiento complejo. Gedisa, Barcelona.

- Morin, E. (1990) Epistemología de la complejidad. "Introducción al pensamiento complejo". Gedisa, Barcelona.

- Morin, E. (1994) Mis demonios. Kairós, Barcelona.

- Morin, E. (1996). Complejidad y teoría social. Centro de Investigaciones SociológicasMadrid

- Morin, E. (2003) El hombre y la muerte. Kairós, Barcelona.

- Morse, J.N. \& Chung, S.E. (2003) Toward holism: The significance of methodologcal pluralism. International Journal of Qualitative Methods, 2/3

- Muntañola, J. (1999) Arquitectura y cultura: nuevos paradigmas. Ediciones UPC, Barcelona.

- Murcia, N; Jaramillo L.J. (2001). La complementariedad como posibilidad. En la estructuración de diseños cualitativos. Index Enfermería.; X (35) 29-33.

- Parse, R. (2000) Paradigms: a reprise. Nurs Sci Q. 2000 Oct;13(4):275-6Ramey, C.H. \& Chrysikou, E.G. (2005) The scientific denial of the real and the dialectic of scientism and
humanism.Am Psychol. 2005;60(4):346-7.

- Peña, K y Rodríguez, J. (2003) La enfermería ante el enfoque del caos y la complejidad. Cultura de los Cuidados. VII/14: 79-82.

- Polit, D. y Hungler, B. (2000). Investigación científica en Ciencias de la Salud ( $5^{\mathrm{a}}$ ed.). México: McGraw-Hill.

- Prigogine, I. (1991) El nacimiento del tiempo. Tusquets, Barcelona.

- Pujadas, J.J. (1992). El método biográfico, las historias de vida en ciencias sociales. C.I.S. Madrid.

- Sandelowski, M. (1999) Time and qualitative research. Res Nurs Health. Feb;22(1):79-87.

- Shambley-Ebron, D.Z. \& Boyle, J.S. (2004)New paradigms for transcultural nursing: frameworks for studying African American women. J Transcult Nurs. 2004 Jan;15(1):11-7.

- Siles, J. et al (1997c) La diferencia entre el aula y el centro sanitario: una apuesta por la implicación del alumnado en el proceso de armonización teórico-práctico (II). Publicación Científica de Enfermería, 10: 17-25

- Siles, J. (1997b ) Epistemología y enfermería. Por una fundamentación científica y profesional de la enfermería. Enfermería Cínica. IV/7: 188-194.

- Siles, J. (2000) Antropología narrativa de los cuidados. Por una rentabilización pedagógica de los materiales narrativos. Una aportación desde la antropología narrativa y la fenomenología. Alicante: Consejo Valenciano de Enfermería.

- Siles, J. Y García, E. (1995) Las características de los paradigmas científicos y su adecuación a la investigación en enfermería. Enfermería Científica, 160/161: 10-15.

- Siles, J., et al. (1997b) Por una rentabilización pedagógica en la obra de Benedetti. Etnología narrativa y situaciones de vida-salud. Cultura de los Cuidados, 1, 17-24.

- Siles, J., et al. (1993) Las alteraciones en la vida cotidiana de los enfermos terminales a través del análisis de textos: Un modelo simulado para las prácticas con el proceso de enfermería. Enfermería Científica, 131, 4-9

- Siles, J; et al (2004b) Las diferencias culturales implicadas en el proceso de prácticas clínicas en enfermería. Una aportación desde la antropología de la complejidad y el pensamiento crítico (II) En: Bernabeu, G y Sauleda, N.. (Edits) Espacios de investigación en la profesionalización docente universitaria. Marfil, Alcoy: 97-117

- Siles, J; et al.(2004a) Antropología educativa aplicada a las prácticas clínicas en enfermería. Las diferencias culturales implicadas en el proceso de practicas clínicas de enfermería. Una aportación desde la antropología de la complejidad y el pensamiento crítico (I).. En: Martínez, M. Coord. Redes de colaboración para el aprendizaje. Marfil, Alcoy: 191-204.

- Skultans, V. (2000). Narrative illnes and the body. Anthropology and Medicine, 7 (1): 5-13

- Solana, J.L (2001) Antropología y complejidad humana : la antropología compleja de Edgar Morin. Comares, Granada

- Solana, J.L. (1999) Reduccionismos antropológicos y antropología compleja. Gazeta de Antropología, 15: 15-09

- Solano, MC; Siles, J (2003). Análisis de las vivencias de un paciente en cuidados intensivos desde la perspectiva de la complejidad. Cultura de los Cuidados, VII (14): 83-91.

- Sontag, S. (1998). El sida y sus metáforas. Barcelona: Muchnik.

- Stockdale, M. \& Warelow P. (2000) Is the complexity of care a paradox? Journal of Advanced Nursing, 31/5: 1258-1264. 
- Stockdale, M. \& Warelow, P. (2000). Is the complexity of care a paradox? Journal of Advanced Nursing, 31/5: 1258-1264.

- Terrel, W. R., Jorgensen, W., \& Wakelin, C. (1992). Human interactive analysis using video: Mapping the dynamics of complex work environments. Educational Technology, 32 (10), 35-41.

- Toffler, A. (1999). "El shock del futuro ". Barcelona: Plaza y Janés.

- Tsuji, Y. (2005) Time is not up: temporal complexity of older Americans' lives.

- J Cross Cult Gerontol. 2005;20(1):3-26.

- Vilar. S. (1997) La nueva racionalidad. Comprender la complejidad con métodos transdisciplinarios. Barcelona: Kairós - Walsh, M. (2000) Chaos, complexity and nursing. Nura Stand.
II, 14/32: 39-42

- Watson, S. (1991) An analysis of concept of experience. Jorunal of Advanced Nursing. 16: 1117-1121.

- Watson, S. (1991). An analysis of concept of experience. Jorunal of Advanced Nursing,16, 1117-1121.

- Wilson Thomas, L. (1995) Applying critical social theory in nursing education to bridge the gap between theory and practice. Journal of Advanced Nursing. 21: 568-575.

- Wilson thomas, L. (1995). Applying critical social theory in nursing education to bridge the gap between theory and practice. Journal of Advanced Nursing, 21, 568-575.

- Yuman, F. (2001) Historia y narración en psicoanálisis. Topía, 33: 11-18

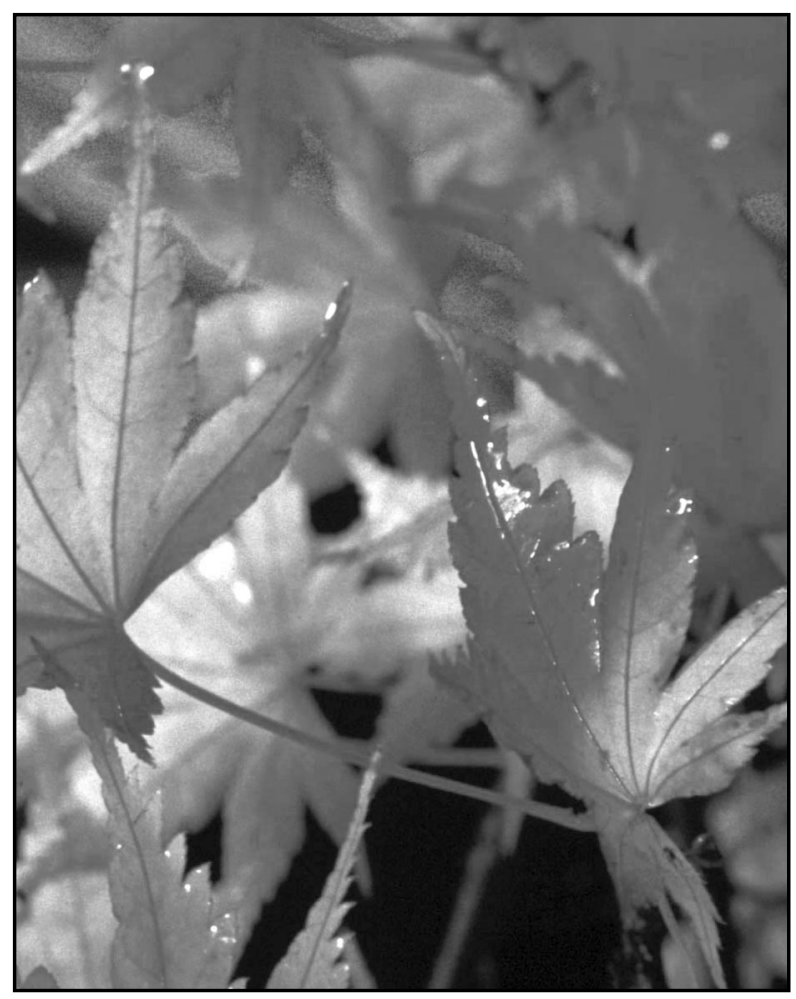




\section{TABLA I \\ EL PARADIGMA \\ TEOLÓGICO}

HECHICERO-CURANDERO-

CHAMÁNBRUJA-SACERDOTE

- AGENTE CONTROLADOR DEL PROCESO.

- MANIPULADOR EXTERNO Y SUBJETIVO RESPECTO DEL ESTADO DE SALUD DEL PACIENTE

- APLICADOR DE PRESCRIPCIONES EN EL TRANSCURSO RITOS MÁGICOS.

- ADMINISTRADOR TERAPÉUTICO DE RITOS MÁGICO-RELIGIOSOS.

PACIENTE

- OBJETO DE CONTROL Y DESTINATARIO DE PRESCRIPCIONES TERAPÉU-

TICAS-MÁGICAS-RELIGIOSAS

CONCEPTO DE

SALUD-ENFERMEDAD

- CONCEPTO SOBRENATURAL

\section{TIPO DE CONOCIMIENTO} CONSTRUIDO

- MAGIA EMPÍRICA MÁGICO RELIGIOSA EXPERIMENTAL

- ANIMISMO Y/O RELIGIONES

- ELABORACIÓN DE CONCEPCIONES DE LA MUERTE EN UN CONTEXTO BIPOLAR:

1) COSMOMORFISMO: INSPIRADO EN EL RECURRENTE RENACIMIENTO DE LA VIDA EN LA NATURALEZA (MUERTE-RESURRECCIÓN O MUERTE-DESCANSO ETERNO: RITOS, RELIGIONES, CELEBRACIONES DE FINALES Y COMIENZOS DE CICLOS).

2) ANTROPOMORFISMO: MANTIENE LA INDIVIDUALIDAD MEDIANTE LA VÍA DE LA INMORTALIDAD: INDIVIDUO AMORTAL.

\section{TABLA II \\ PARADIGMA \\ TECNOLÓGICO}

\section{PROFESIONAL TÉCNICO}

DE LA SALUD

- AGENTE CONTROLADOR DEL PROCESO.

- MANIPULADOR EXTERNO Y OBJETIVO RESPECTO DEL ESTADO DE SALUD

PACIENTE Y SUS NECESIDADES

- APLICADOR DE PRESCRIPCIONES.

CONCEPTO DE SALUD

- CONCEPTO NEGATIVO/VACÍO: SALUD COMO AUSENCIA DE ALGO, OMISIÓN DE MANIFESTACIONES CLÍNICAS O PRIVACIÓN DE SINTOMATOLOGÍA.

\section{TIPO DE CONOCIMIENTO CONSTRUIDO}

- CIENCIA NATURAL HEREDERA DEL NEOPOSITIVISMO

- CARÁCTER OBJETIVO DE LA ACTIVIDAD CIENTÍFICA

- CONTROL EXTERNO DEL PROCESO.

¿CÓMO SE CONSTRUYe?

- INVESTIGACIÓN EXPERIMENTAL (PRUEBAS ESTANDARIZADAS, TEST, ENCUESTAS).

\section{CONCEPTOS DE ENFERMEDAD Y MUERTE}

- ELABORACIÓN CIENTÍFICO TÉCNICA DEL CONCEPTO ENFERMEDAD Y MUERTE 


\section{TABLA III \\ PARADIGMA HERMENEUTICO}

PROFESIONAL DE LA SALUD

- AGENTE DE COMUNICACIÓN COMPRENSIVA E INTERACTIVA.

- INTERPRETADOR DE LA REALIDAD DEL PACIENTE

\section{CONCEPTO DE SALUD}

- CONCEPTO SITUACIONAL ELABORADO INTERACTIVAMENTE (PACIENTEENFERMERO)

\section{TIPO DE CONOCIMIENTO CONSTRUIDO}

- ENFERMERÍA COMO CONSTRUCCIÓN PERSONAL

- SOCIOLOGÍA INTERACCIONISTA.

- HERMENÉUTICA Y FENOMENOLOGÍA DE LOS CUIDADOS.

- ANTROPOLOGÍA CULTURAL.

\section{¿CÓMO SE CONSTRUYE?}

- INVESTIGACIÓN ETNOGRÁFICA.

- ESTUDIO DE SIGNIFICADOS EN SUS CONTEXTOS (USO DE MÉTODOS Y TÉCNICAS FENOMENOLÓGICAS, NARRATIVAS, MATERIAL BIOGRÁFICO Y AUTOBIOGRÁFICO)

\section{CONCEPTO DE ENFERMEDAD Y MUERTE}

- RESULTADO DE ÑA INTERVENCIÓN PRÁCTICA INTERPRETATIVA. ACTO DE COMPRENSIÓN INDIVIDUAL

- ENFERMEDAD Y MUERTE INTERPRETADOS INDIVIDUALMENTE SEGÚN CREENCIAS, VALORES, SITUACIÓN PERSONA.

- NO EXISTE UN CONCEPTO EXCLUSIVO DE ENFERMEDAD Y NUERTE, RESPONDE A REALIDADES SITUACIONALES

\section{TABLA IV \\ PARADIGMA SOCIOCRÍTICO}

\section{PROFESIONA DE LA SALUD}

- AGENTE DE CAMBIO SOCIOSANITARIO

\section{CONCEPTO DE SALUD}

- CONCEPTO POSITIVO.

- REELABORACIÓN DEL MISMO POR PARTE DEL SUJETO.

\section{TIPO DE CONOCIMIENTO CONSTRUIDO}

-ENFERMERÍA COMO CONSTRUCCIÓN SOCIAL Y SANITARIA.

-DIALÉCTICA-SUBJETIVIDAD

-CONSTRUCCIÓN NUEVAS REALIDADES.

\section{¿CÓMO SE CONSTRUYE?}

-INVESTIGACIÓN CRÍTICA -ESTUDIOS DE CONTEXTOS PERSONALES Y SOCIALES.

\section{¿PARA QUÉ SIRVE?}

-ENFERMERIA ENFOCADA COMO INTERVENCIÓN EN PRÁCTICA SOCIAL Y ANTROPOLÓGICA DEL SUJETO.

\section{CONCEPTOS DE ENFERMEDAD Y MUERTE}

TEORÍA MULTIDIMENSIONAL BASADA "RADICALES ANTROPOLÓGICOS O ANTROPOCOSMOLÓGICOS". (MORIN)

* PRODUCCIÓN (MARX)

* PSIQUE (FREUD)

* EL AMOR (CRISTIANISMO)

* LA CIENCIA MODERNA

* LA POESÍA (REIVINDICADA POR EL SUPERREALISMO) 